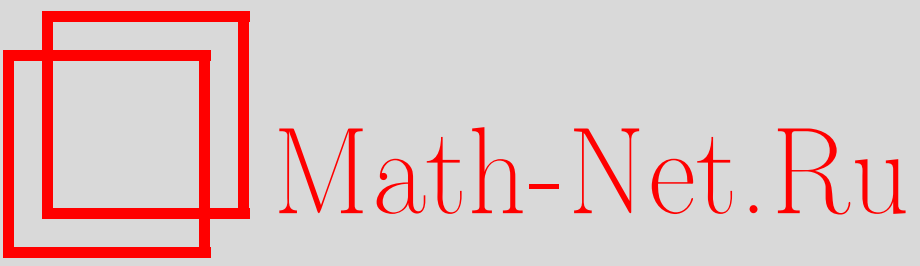

Г. Э. Арутюнов, Построение классических тригонометрических $r$-матриц модели Тоды методом гамильтоновой редукции кокасательных расслоений над группами петель, ТМФ, 1997, том 113, номер 1, 3-12

DOI: https://doi.org/10.4213/tmf1060

Использование Общероссийского математического портала Math-Net.Ru подразумевает, что вы прочитали и согласны с пользовательским соглашением

http://www.mathnet.ru/rus/agreement

Параметры загрузки:

IP : 54.205 .225 .156

26 апреля 2023 г., 13:39:55 
ТЕОРЕТИЧЕСКАЯ

И МАТЕМАТИЧЕСКАЯ

ФИЗИКА

Том 113, № 1

октябрь, 1997

\section{Г.Э. Арутюнов* \\ ПОСТРОЕНИЕ КЛАССИЧЕСКИХ ТРИГОНОМЕТРИЧЕСКИХ $r$-МАТРИЦ МОДЕЛИ ТОДЫ МЕТОДОМ ГАМИЛЬТОНОВОЙ РЕДУКЦИИ КОКАСАТЕЛЬНЫХ РАССЛОЕНИЙ НАД ГРУППАМИ ПЕТЕЛЬ}

\footnotetext{
Показано, как классические тригонометрические $r$-матрицы модели Тоды возникают в результате гамильтоновой редукции кокасательных расслоений над группами петель.
}

\section{1. ВВЕДЕНИЕ}

Цель настоящей работы - показать, как известные тригонометрические $r$-матрицы модели Тоды $[1,2]$ могут быть получены методом гамильтоновой редукции, примененным к кокасательному расслоению над группой петель.

Хорошо известно, что конечномерные динамические системы типа Тоды и Калоджеро-Мозера-Сазерленда получаются соответствуюшей гамильтоновой редукцией инвариантных гамильтонианов, заданных на кокасательных расслоениях простых групा Ли $[3,4]$. $L$-оператор, участвуюший в представлении Лакса, возникает при этом как функция на редуцированном фазовом пространстве $\mathcal{P}$, а само представление Лакса $d L / d t=[M, L]-$ как уравнение движения на $\mathcal{P}$. Помимо того что такая конструкция придает $L$-оператору геометрический смысл, она также позволяет вычислить классическую $r$-матрицу (при условии, что она сушествует), т.е. доказать тем самым кинематическую интегрируемость изучаемой системы. Недавно этот подход был применен для вычисления динамических $r$-матриц модели Калоджеро-Мозера-Сазерленда [5]. Заметим, что рассматриваемые $L$-операторы не содержат спектрального параметра, который необходим для построения переменных действие-угол. Таким образом, встает вопрос о рассмотрении такой схемы гамильтоновой редукции, в которой бы возникало представление Лакса динамической системы со спектральным параметром.

Данная работа представляет собой перенесение конструкции [5] на аффинный случай. Мы покажем на примере модели Тоды, что представление Лакса со спектральным параметром можно получить, рассматривая гамильтонову редукцию кокасательного расслоения над группой (скрученных) петель. При этом петлевой параметр естественным

* Математический институт им. В. А. Стеклова, Москва, Россия. E-mail: arut@genesis.mi.ras.ru 
образом отождествляется со спектральным параметром. Важная особенность рассматриваемой редукции заключается в том, что редуцированное фазовое пространство оказывается конечномерным. $r$-Матрищы вычисляются так же, как в [5], и оказьваются тригонометрическими решениями классического уравнения Янга-Бакстера.

Отметим, что само утверждение о связи тригонометрических $r$-матриц с аффинными алгебрами хорошо известно (см., например, [6]). Как будет следовать из нашего рассмотрения, с точки зрения гамильтоновой редукции эта связь обусловлена наличием разложения Ивасавы для бесконечномерной группы петель. Для упрошения изложения мы ограничиваемся рассмотрением аффинных алгебр высоты 1.

\section{2. АФФИННЫЕ АЛГЕБРЫ И РАЗЛОЖЕНИЕ ИВАСАВЫ}

Пусть $\widehat{\mathcal{G}}$ обозначает алгебру Каца-Муди высоты 1 ранга $l$. Известно [7], что фактор-алгебра $\mathcal{G}=\widehat{\mathcal{G}} / \mathbf{C} c$ алгебры $\widehat{\mathcal{G}}$ по ее одномерному центру с имеет две основные реализащии - стандартную и каноническую, возникающие из соответствуюших реализаций $\widehat{\mathcal{G}}$.

Пусть $A_{i j}$ - матрица Картана алгебры $\widehat{\mathcal{G}}$, а $a_{i}$ - отметки диаграммы Дынкина. Напомним, что как абстрактная алгебра $\mathcal{G}$ порождается $3(l+1)$ образующими $\left\{e_{i}, h_{i}, f_{i}\right\}$, $i=0, \ldots, l$, и соотношениями

$$
\begin{gathered}
{\left[h_{i}, h_{j}\right]=0, \quad\left[e_{i}, f_{j}\right]=\delta_{i j} h_{i},} \\
{\left[h_{i}, e_{j}\right]=A_{i j} e_{j}, \quad\left[h_{i}, f_{j}\right]=-A_{i j} f_{j},} \\
\left(\operatorname{ad} e_{i}\right)^{1-A_{i j}} e_{j}=\left(\operatorname{ad} f_{i}\right)^{1-A_{i j}} f_{j}=0,
\end{gathered}
$$

а также дополнительным соотношением $\sum_{i=0}^{l} a_{i} h_{i}=0$, фиксируюшим центральный элемент $c=\sum_{i=0}^{l} a_{i} h_{i}$ алгебры (1) равным нулю. $\mathcal{G}$ является градуированной алгеброй Ли.

1. Стандартная градуировка $\mathcal{G}$. Положим $\operatorname{deg} e_{i}=\operatorname{deg} f_{i}=\operatorname{deg} h_{i}=0, i=$ $1, \ldots, l$, и $\operatorname{deg} e_{0}=-\operatorname{deg} f_{0}=1$, где тройка образуюших $e_{0}, h_{0}, f_{0}$ соответствует специальной вершине диаграммы Дынкина. Нетрудно показать, что градуированная таким образом алгебра $\mathcal{G}$ изоморфна алгебре петель $\overline{\mathcal{G}}\left[\lambda, \lambda^{-1}\right]$ над простой конечномерной алгеброй Ли $\overline{\mathcal{G}}$, полученной из $\widehat{\mathcal{G}}$ вычеркиванием специальной вершины диаграммы Дынкина: $\mathcal{G}=\sum_{k \in \mathbf{Z}} \overline{\mathcal{G}} \otimes \lambda^{k}$. Заметим, что пространство $\mathcal{G}^{k}=\overline{\mathcal{G}} \otimes \lambda^{k}$ линейно порождается элементами

$$
E_{\alpha}^{k}=e_{\alpha} \otimes \lambda^{k}, \quad h_{i}^{k}=h_{i} \otimes \lambda^{k}, \quad i=1, \ldots, l,
$$

где $\alpha \in \Delta, \Delta$ - множество корней $\overline{\mathcal{G}}$.

2. Каноническая градуировка $\mathcal{G}$. Каноническая градуировка $\mathcal{G}=\sum_{k \in \mathbf{Z}} \mathcal{G}^{k}$ задается следующим образом: $\operatorname{deg} e_{i}=-\operatorname{deg} f_{i}=1, i=0, \ldots, l$, и $\operatorname{deg} h_{i}=0$. Можно показать, что при таком выборе градуировки $\mathcal{G}$ изоморфна алгебре скрученных петель $\mathcal{G}=\sum_{k \in \mathbf{Z}} \overline{\mathcal{G}}_{k \bmod h} \otimes \lambda^{k}$, построенной с помощью разложения $\overline{\mathcal{G}}$ по собственным значениям кокстеровского автоморфизма $C$ :

$$
\overline{\mathcal{G}}_{k}=\left\{x \in \overline{\mathcal{G}}, \quad C(x)=\omega^{k} x, \quad \omega=e^{\frac{2 \pi i}{h}}\right\},
$$

где $h$ - число Кокстера для $\widehat{\mathcal{G}}$. 
Выберем теперь в $\mathcal{G}$ линейный базис однородных относительно канонической градуировки элементов. Если $k \neq 0 \bmod h$, то пространство $\mathcal{G}^{k}$ линейно порождается элементами $E_{\alpha}^{k}=e_{\alpha} \otimes \lambda^{k}$, где $\alpha \in \Delta^{k}$ и $\Delta^{k}=\left\{\alpha \in \Delta, e_{\alpha} \in \overline{\mathcal{G}}_{k}\right\}$, т.е. $e_{\alpha}$ пробегает множество всех корневых векторов, лежаших в $\overline{\mathcal{G}}_{k}$. Если же $k=0 \bmod h$, то векторы $h_{i}^{k}=h_{i} \otimes \lambda^{k}$ составляют базис в $\mathcal{G}^{k}$. Итак,

$$
\mathcal{G}=\sum_{k \neq 0 \bmod h} \sum_{\alpha \in \Delta^{k \bmod h}} \mathbf{C} E_{\alpha}^{k}+\sum_{k} \mathbf{C} h_{i}^{k h} .
$$

Для дальнейшего удобно ввести следующие обозначения. Обозначим через $n_{+}\left(n_{-}\right)$ подалгебру в $\mathcal{G}$, порожденную как векторное пространство кратными коммутаторами элементов $e_{i}\left(f_{i}\right), 0 \leq i \leq l$, а через $\bar{h}$ - картановскую подалгебру в $\overline{\mathcal{G}}$.

ПрЕДЛОЖениЕ 1. Пусть алгебра $\mathcal{G}$ градуирована стандартныцм образом, тогда

$$
\begin{aligned}
& n_{+}=\left(\bar{n}_{+} \otimes 1\right) \oplus(\overline{\mathcal{G}} \otimes \lambda \mathbf{C}[\lambda]), \\
& n_{-}=\left(\bar{n}_{-} \otimes 1\right) \oplus\left(\overline{\mathcal{G}} \otimes \lambda^{-1} \mathbf{C}\left[\lambda^{-1}\right]\right),
\end{aligned}
$$

где $\bar{n}_{ \pm}-$нильпотентные подалгебры в треугольном разложении $\overline{\mathcal{G}}=\bar{n}_{+} \oplus \bar{h} \oplus \bar{n}_{-}$.

ДокАЗАтЕльСТво. Образуюшие Шевалле (1) для $\mathcal{G}$ можно выбрать следуюшим образом. Выберем в $\overline{\mathcal{G}}$ вектор $\bar{e}_{0}\left(\bar{f}_{0}\right)$, соответствуюший старшему (младшему) корню, и нормируем его условием $\left(\bar{e}_{0}, \bar{f}_{0}\right)=1$. Обозначим через $\bar{e}_{i}\left(\bar{f}_{i}\right), 1 \leq i \leq l$, корневые векторы, отвечающие простым положительным (отрицательным) корням и коммутируюшие как $\left[\bar{e}_{i}, \bar{f}_{i}\right]=\bar{h}_{i}$. Тогда генераторы Шевалле для $\mathcal{G}$ даются векторами

$$
e_{0}=\bar{f}_{0} \otimes \lambda, \quad f_{0}=\bar{e}_{0} \otimes \lambda^{-1}, \quad h_{0}=\left[e_{0}, f_{0}\right]
$$

вместе с $\left\{\bar{e}_{i}, \bar{h}_{i}, \bar{f}_{i}\right\}_{1 \leq i \leq l}$. Рассматривая теперь кратные коммутаторы генераторов $e_{i}$, получаем требуемое утверждение. Реализация образуюших Шевалле, приведенная в предложении 1 , называется стандартной.

Пусть $\mathcal{G}$ градуирована стандартным образом. Обозначим через $\mathcal{G}^{R}$ вешественную нормальную форму $\mathcal{G} . \mathcal{G}^{R}$ порождается базисом $E_{\alpha}^{k}=e_{\alpha} \otimes \lambda^{k}, h_{i}^{k}=h_{i} \otimes \lambda^{k}$ над полем $\mathbf{R}$. Рассмотрим в $\mathcal{G}^{R}$ инволюцию $\tau$, задаваемую на образуюших формулами

$$
\tau\left(E_{\alpha}^{k}\right)=-E_{-\alpha}^{-k}, \quad \tau\left(h_{i}^{k}\right)=-h_{i}^{-k} .
$$

ПреДЛОЖенИЕ 2. Алгебра $\mathcal{K}$ неподвижных точек инволюиии $\tau$ в $\mathcal{G}^{R}$ порождается линейным базисом

$$
\mathcal{K}=\sum_{\substack{\alpha \in \Delta \\ k>0}} \mathbf{R} V_{\alpha}^{k}+\sum_{\alpha \in \Delta_{+}} \mathbf{R} V_{\alpha}^{0}+\sum_{\substack{1 \leq i \leq l \\ k>0}} \mathbf{R} H_{i}^{k}
$$

əде $V_{\alpha}^{k}=E_{\alpha}^{k}-E_{-\alpha}^{-k}$ u $H_{i}^{k}=h_{i}^{k}-h_{i}^{-k}$. 
ДоКАЗАТЕЛЬСтво предложения тривиально.

На алгебре $\mathcal{G}^{R}$ сушествует невырожденная симметрическая билинейная форма, инвариантная относительно присоединенного действия. Нетрудно видеть, что эта форма отрицательно определена на $\mathcal{K}$, т.е. $\mathcal{K}$ является компактной подалгеброй в $\mathcal{G}^{R}$. Таким образом, $\mathcal{G}^{R}$ распадается в прямую сумму $\mathcal{G}^{R}=\mathcal{K} \oplus \mathcal{M}$ алгебры $\mathcal{K}$ и векторного пространства $\mathcal{M}$ :

$$
\mathcal{M}=\sum_{\substack{\alpha \in \Delta \\ k>0}} \mathbf{R} W_{\alpha}^{k}+\sum_{\alpha \in \Delta_{+}} \mathbf{R} W_{\alpha}^{0}+\sum_{\substack{1 \leq i \leq l \\ k \geq 0}} \mathbf{R} T_{i}^{k}
$$

где $W_{\alpha}^{k}=E_{\alpha}^{k}+E_{-\alpha}^{-k}$ и $T_{i}^{k}=h_{i}^{k}+h_{i}^{-k}$. Указанное разложение является аналогом разложения Картана для полупростой конечномерной алгебры Ли над полем $\mathbf{R}$.

В дальнейшем важную роль будет играть аналог разложения Ивасавы. Заметим, что любой вектор $x \in \mathcal{G}$ представим в виде

$$
\begin{aligned}
x= & \sum_{\substack{\alpha \in \Delta \\
k>0}} a_{\alpha}^{k}\left(E_{\alpha}^{k}-E_{-\alpha}^{-k}\right)+\sum_{\alpha \in \Delta_{+}} a_{\alpha}^{0}\left(E_{\alpha}^{0}-E_{-\alpha}^{0}\right)+\sum_{\substack{1 \leq i \leq l \\
k>0}} a_{i}^{k}\left(h_{i}^{k}-h_{i}^{-k}\right)+ \\
& +\sum_{\substack{\alpha \in \Delta \\
k>0}} b_{\alpha}^{k}\left(E_{\alpha}^{k}+E_{-\alpha}^{-k}\right)+\sum_{\alpha \in \Delta_{+}} b_{\alpha}^{0}\left(E_{\alpha}^{0}+E_{-\alpha}^{0}\right)+\sum_{\substack{1 \leq i \leq l \\
k \geq 0}} b_{i}^{k}\left(h_{i}^{k}+h_{i}^{-k}\right) .
\end{aligned}
$$

Это разложение можно перегруппировать следующим образом:

$$
\begin{aligned}
x= & \sum_{\substack{\alpha \in \Delta \\
k>0}}\left(a_{\alpha}^{k}-b_{\alpha}^{k}\right) V_{\alpha}^{k}+\sum_{\alpha \in \Delta_{+}}\left(a_{\alpha}^{0}-b_{\alpha}^{0}\right) V_{\alpha}^{0}+\sum_{\substack{1 \leq i \leq l \\
k>0}}\left(a_{i}^{k}-b_{i}^{k}\right) H_{i}^{k}+ \\
& +\sum_{\substack{\alpha \in \Delta \\
k>0}} 2 b_{\alpha}^{k} E_{\alpha}^{k}+\sum_{\alpha \in \Delta_{+}} 2 b_{\alpha}^{0} E_{\alpha}^{0}+\sum_{\substack{1 \leq i \leq l \\
k>0}} 2 b_{i}^{k} h_{i}^{k}+\sum_{1 \leq i \leq l} 2 b_{i}^{0} h_{i} .
\end{aligned}
$$

В силу предложения 1 элемент

$$
\sum_{\substack{\alpha \in \Delta \\ k>0}} 2 b_{\alpha}^{k} E_{\alpha}^{k}+\sum_{\alpha \in \Delta_{+}} 2 b_{\alpha}^{0} E_{\alpha}^{0}+\sum_{\substack{1 \leq i \leq l \\ k>0}} 2 b_{i}^{k} h_{i}^{k}
$$

лежит в $n_{+}$. Следовательно, $\mathcal{G}^{R}$ распадается в прямую сумму трех подалгебр: $\mathcal{G}^{R}=\mathcal{K} \oplus$ $n_{+} \oplus a$, где $a$ - вложение $\bar{h}$ в $\mathcal{G}^{R}$. Это разложение будем назьвать разложением Ивасавы для $\mathcal{G}^{R}$. Разложение Ивасавы имеет глобальный аналог $G=N_{+} A K$, в котором $G$ обозначает группу петель, а $N_{+}, A$ и $K$ - односвязные группы Ли с алгебрами $n_{+}, a$ и $\mathcal{K}$, соответственно. Отметим, что $N_{+}$можно рассматривать как группу, состоящую из граничных значений голоморфных отображений

$$
\gamma:\{z:|z|<1\} \rightarrow \overline{\mathcal{G}}^{R}
$$

таких, что $\gamma(0)$ лежит в нильпотентной подгруппе группы $\overline{\mathcal{G}}^{R}$, отвечающей положительным корням [8]. 
Рассмотрим теперь разложение Картана для канонической реализации $\mathcal{G}$. Инволюция задается той же формулой (4), поэтому собственные подпространства автоморфизма $\tau$ в $\mathcal{G}=\sum_{k \in \mathbf{Z}} \overline{\mathcal{G}}_{k}(\bmod h) \otimes \lambda^{k}$ имеют вид

$$
\mathcal{K}=\sum_{\substack{k>0 \\ k \neq 0 \bmod h}} \sum_{\alpha \in \Delta^{k \bmod h}} \mathbf{R} V_{\alpha}^{k}+\sum_{\substack{k>0 \\ 1 \leq i \leq l}} \mathbf{R} H_{i}^{k h}
$$

и

$$
\mathcal{M}=\sum_{\substack{k>0 \\ k \neq 0 \bmod h}} \sum_{\alpha \in \Delta^{k \bmod h}} \mathbf{R} W_{\alpha}^{k}+\sum_{\substack{k \geq 0 \\ 1 \leq i \leq l}} \mathbf{R} T_{i}^{k h}
$$

Как и для стандартной реализации, из этих представлений немедленно вытекает разложение Ивасавы для алгебры петель, скрученных с помощью кокстеровского автоморфизма.

\section{3. ГАМИЛЬТОНОВА РЕДУКЦИЯ КОКАСАТЕЛЬНОГО РАССЛОЕНИЯ НАД ГРУППОЙ ЛИ}

Пусть $G$ - группа Ли с алгеброй Ли $\mathcal{G}$, а $T^{*} G$ - кокасательное расслоение над $G$. Используя левые сдвиги, можно отождествить $T^{*} G$ с $G \times \mathcal{G}^{*}$, где $\mathcal{G}^{*}$ есть дуальное пространство к $\mathcal{G}$. Таким образом, точка $T^{*} G$ - это пара $(g, \xi)$, где $g \in G$ и $\xi \in \mathcal{G}^{*}$. Каноническая пуассонова структура на $T^{*} G$ имеет вид

$$
\{\phi, \psi\}=\{\phi, \psi\}_{\mathrm{K}}+\partial^{i} \phi L_{i} \psi-\partial^{i} \psi L_{i} \phi, \quad \phi, \psi \in \operatorname{Fun}\left(T^{*} G\right)
$$

Поясним обозначения в последней формуле. Выберем в пространствах $\mathcal{G}$ и $\mathcal{G}^{*}$ дуаль-

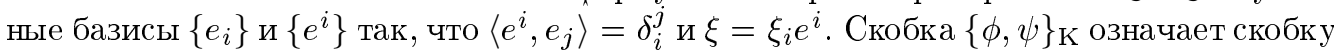
Кириллова ограничения функций $\phi$ и $\psi$ на $\mathcal{G}^{*}$ :

$$
\{\phi, \psi\}_{\mathrm{K}}=f_{i j}^{k} \xi_{k} \partial^{i} \phi \partial^{j} \psi
$$

где $f_{i j}^{k}$ - структурные константы $\mathcal{G}$ в базисе $\left\{e_{i}\right\}$ и $\partial^{i} \phi=\left(\partial / \partial \xi_{i}\right) \phi$. Оператор $L_{i}$ рассматривается как левоинвариантное векторное поле на $G$, порожденное элементом $e_{i}$ :

$$
\left(L_{i} \phi\right)(g)=\left.\frac{d}{d t}\right|_{t=0} \phi\left(g e^{t e_{i}}\right) .
$$

Группа $G$ действует на $T^{*} G$ левыми и правыми сдвигами:

$$
g^{\prime} \cdot(g, \xi)=\left(g^{\prime} g, \xi\right), \quad(g, \xi) \cdot g^{\prime}=\left(g\left(g^{\prime}\right)^{-1}, \operatorname{Ad}_{g^{\prime}}^{*} \xi\right)
$$

Эти действия порождают фундаментальные векторные поля на $T^{*} G$, являющиеся гамильтоновыми. Соответствующие гамильтонианы имеют вид

$$
H_{X}^{L}(g, \xi)=\left\langle\xi, g^{-1} X g\right\rangle, \quad H_{X}^{R}(g, \xi)=-\langle\xi, X\rangle, \quad X \in \mathcal{G}
$$


В самом деле, из (9) получаем

$$
\left\{H_{X}^{R}, \psi\right\}=\left\langle\operatorname{ad}_{X}^{*} \xi, \nabla \psi\right\rangle-L_{X} \psi=\left.\frac{d}{d t}\right|_{t=0} \psi\left(g e^{-t X}, \operatorname{Ad}_{e^{t X}}^{*} \xi\right),
$$

где $\nabla \psi=e_{i} \partial^{i} \psi$. Аналогичное соотношение можно записать и для $H_{X}^{L}$.

Пусть подгруппы $\mathcal{H}^{L}$ и $\mathcal{H}^{R}$ группы $G$ с алгебрами Ли $h^{L}$ и $h^{R}$ действуют на $T^{*} G$ левыми и правыми сдвигами, соответственно. В силу сказанного вьше эти действия гамильтоновы, а соответствующие отображения момента $\mu^{L}: T^{*} G \rightarrow\left(h^{L}\right)^{*}$ и $\mu^{R}: T^{*} G \rightarrow\left(h^{R}\right)^{*}$ даются формулами

$$
\begin{array}{ll}
\left\langle\mu^{L}(g, \xi), X\right\rangle=\left\langle\xi, g^{-1} X g\right\rangle, & X \in h^{L}, \\
\left\langle\mu^{R}(g, \xi), X\right\rangle=-\langle\xi, X\rangle, & X \in h^{R} .
\end{array}
$$

Рассмотрим редукцию $T^{*} G$ по действию группы $\mathcal{H}=\left(\mathcal{H}^{L}, \mathcal{H}^{R}\right)$. Согласно общей схеме гамильтоновой редукции [9] фактор-пространство

$$
\mathcal{P}_{\mu}=\mu^{-1}\left(T^{*} G\right) / \operatorname{Stab} \mu
$$

уровня постоянного момента $\mu=$ const по его стабилизатору Stab $\mu$ в $\mathcal{H}$ снабжается при некоторых дополнительных условиях симплектической структурой. Соответствующую скобку Пуассона $\{,\}_{\mathcal{P}_{\mu}}$ можно рассчитать, используя множество $\operatorname{Fun}\left(T^{*} G\right)^{\mathcal{H}}$ $\mathcal{H}$-инвариантных функций на $T^{*} G$, а именно,

$$
\{\phi, \psi\}_{\mathcal{P}_{\mu}}=\left.\{\phi, \psi\}\right|_{\mu=\mathrm{const}}, \quad \phi, \psi \in \operatorname{Fun}\left(T^{*} G\right)^{\mathcal{H}} .
$$

Пусть теперь $G$ есть группа вешественных (скрученных) петель, а $G=N_{+} A K-$ ее разложение Ивасавы, описанное в предыдущем разделе. Положим $\mathcal{H}^{L}=N_{+}$и $\mathcal{H}^{R}=K$, а также выберем по аналогии с конечномерной ситуацией $[4,5] \mu^{R}=0$ и $\mu^{L}=I=$ $\sum_{i=0}^{l} f_{i} \in n_{-}=\left(n_{+}\right)^{*}$.

ПреДЛОЖенИЕ 3. Стабилизатор әлемента I совпадает с $N_{+}$.

ДокАзАтЕЛьСтво. Алгебра Ли Stab $I$ определяется условием

$$
\langle I,[X, Y]\rangle=0, \quad X, Y \in \mathcal{L} i e(\operatorname{Stab} I),
$$

где $\langle$,$\rangle означает билинейную симметрическую форму на \mathcal{G}$, согласованную как со стандартной, так и с канонической градуировкой. Покажем, что если $X, Y \in n_{+}$, то (15) выполнено.

Пусть $\mathcal{G}$ градуирована стандартным образом. Для любых однородных элементов $X, Y \in n_{+}$имеем $\operatorname{deg} X, \operatorname{deg} Y \geq 0$, так что $\operatorname{deg}[X, Y] \geq 0$. Поскольку $I \in \mathcal{G}^{-1} \oplus \mathcal{G}^{0}$ и форма $\langle$,$\rangle согласована со стандартной градуировкой, т.е. \left\langle\mathcal{G}^{k}, \mathcal{G}^{m}\right\rangle=\delta_{k,-m}$, то возникают два нетривиальных случая:

a) $[X, Y] \in \bar{n}_{+}$, т.е. этот коммутатор порожден элементами $e_{i}, 1 \leq i \leq l$, и потому не содержит в своем разложении по корневому базису в $\overline{\mathcal{G}}$ ни одного простого корня, отсюда следует (15); 
б) $[X, Y] \in \mathcal{G}^{1}$, т.е. один из элементов, скажем $X$, содержит один генератор $e_{0}$; тогда $\left\langle f_{0},[X, Y]\right\rangle=-\left\langle\left[X, f_{0}\right], Y\right\rangle$. Замечая, что $\left[X, f_{0}\right] \in \bar{n}_{+}$, и пользуясь изотропностью $\bar{n}_{+}$, получаем (15).

Случай канонической градуировки рассматривается аналогично. Предложение 3 доказано.

Из предложения 3 следует, что стабилизатор выбранного нами момента совпадает с группой $N_{+} \times K$. Используя эту симметрию, любую точку $(g, \xi) \in T^{*} G$, лежашую на поверхности уровня данного момента, можно привести к виду $(Q, L)$, где $Q \in A$ в разложении Ивасавы. Пара $(Q, L)$ задает, по сути, координаты на фактор-пространстве $\mathcal{P}_{\mu}$. Вид $L$ фиксируется из условия принадлежности $(Q, L)$ поверхности $\mu^{R}=0, \mu^{L}=I$ :

$$
\langle L, X\rangle=0 \text { для } \forall X \in \mathcal{K}, \quad\left\langle Q^{-1} L Q, X\right\rangle=\langle I, X\rangle \text { для } \forall X \in n_{+} .
$$

В силу ортогональности $\mathcal{K}$ и $\mathcal{M}$ первое из этих уравнений дает

$$
\begin{aligned}
L^{\text {st }} & =\sum_{\substack{k \geq 0 \\
1 \leq i \leq l}} a_{i}^{k}\left(h_{i}^{k}+h_{i}^{-k}\right)+\sum_{\substack{\alpha \in \Delta \\
k \geq 0}} a_{\alpha}^{k}\left(E_{\alpha}^{k}+E_{-\alpha}^{-k}\right), \\
L^{\mathrm{can}} & =\sum_{\substack{k \geq 0 \\
1 \leq i \leq l}} a_{i}^{k}\left(h_{i}^{k h}+h_{i}^{-k h}\right)+\sum_{\substack{k>0, k \neq 0 \bmod h \\
\alpha \in \Delta^{k \bmod h}}} a_{\alpha}^{k}\left(E_{\alpha}^{k}+E_{-\alpha}^{-k}\right) .
\end{aligned}
$$

Здесь $L^{\mathrm{st}}$ и $L^{\text {can }}$ обозначают $L$ в стандартной и канонической градуировках, соответственно. Поскольку $A$ в разложении Ивасавы совпадает с группой постоянны $x$ петель со значениями в эспоненцированной $\bar{h}$, то $Q=e^{q}, q \in \bar{h}$. Подставляя теперь эти $L$ во второе уравнение (16), которое можно переписать в виде $P_{n_{-}}\left(e^{-q} L e^{q}\right)=I\left(P_{n_{-}}\right.$есть проектор на $\left.n_{-}\right)$, а также используя тождества $e^{-q} E_{ \pm \alpha}^{k} e^{q}=e^{\mp \alpha(q)} E_{ \pm \alpha}^{k}$, получаем в обоих случаях одно и то же формальное выражение для $L$ :

$$
L=\sum_{1 \leq i \leq l} \pi_{i} h_{i}+\sum_{1 \leq i \leq l} e^{-\alpha_{i}(q)}\left(e_{i}+f_{i}\right)
$$

Построенный таким образом оператор $L$ хорошо известен как $L$-оператор цепочки Тоды [10]. Выбирая матричное представление для $\overline{\mathcal{G}}$ и подставляя в (19) стандартную или каноническую реализацию образуюших Шевалле, мы получаем $L$-оператор цепочки Тоды как матричнозначную функцию спектрального параметра $\lambda$.

\section{4. КЛАССИЧЕСКИЕ $r$-МАТРИЦЫ ЦЕПОЧКИ ТОДЫ}

Здесь мы покажем, как, используя пуассонову структуру на редуцированном фазовом пространстве, можно вычислить $r$-матрищы цепочки Тоды.

Сначала рассмотрим конечномерную ситуацию. Пусть $g=n Q k$ - разложение Ивасавы вещественной полупростой группы Ли. Функция $F_{X}(Q, L), X \in \mathcal{M}$, заданная на $\mathcal{P}_{\mu}$, имеет расширение $F_{X}(g, \xi)=\left\langle\xi, k^{-1} X k\right\rangle$ на $T^{*} G$, инвариантное относительно действия группы $\mathcal{H}=N_{+} \times K$. В работе[5] доказано следующее предложение.

ПрЕДЛОЖЕНИЕ 4. СКобка Пуассона на редуцированном пространстве имеет вид

$$
\left\{F_{X}, F_{Y}\right\}(Q, L)=\langle L,[r(X), Y]+[X, r(Y)]\rangle,
$$


әде линейный оператор $r: \mathcal{M} \rightarrow \mathcal{K}$ задается формулой

$$
r(X)=\left.\frac{d}{d t}\right|_{t=0} k\left(g e^{t X}\right) .
$$

Отметим, что (20) следует непосредственно из (14).

Записывая для элемента $X \in \mathcal{M}$ разложение Ивасавы в виде $X=X_{+}+X_{a}+X_{k}$, где $X_{+} \in n_{+}, X_{a} \in a, X_{k} \in \mathcal{K}$, несложно найти [5], что

$$
r(X)=-X_{k}
$$

Очевидно, что эта формула без изменений переносится на рассматриваемый нами случай редукции бесконечномерной группы.

1. $r$-Матрица в стандартной градуировке. Подставляя разложение произвольного элемента $x$ по однородному базису в уравнение (21):

$$
\begin{gathered}
r\left(\sum_{\substack{\alpha \in \Delta \\
n>0}} x_{\alpha}^{n} W_{\alpha}^{n}+\sum_{\alpha \in \Delta_{+}} x_{\alpha}^{0} W_{\alpha}^{0}+\sum_{\substack{1 \leq i \leq l \\
n \geq 0}} x_{i}^{n} T_{i}^{n}\right)= \\
=-\sum_{\substack{\alpha \in \Delta \\
n>0}} x_{\alpha}^{n} V_{\alpha}^{n}+\sum_{\alpha \in \Delta_{+}} x_{\alpha}^{0} V_{\alpha}^{0}+\sum_{\substack{1 \leq i \leq l \\
n>0}} x_{i}^{n} H_{i}^{n}
\end{gathered}
$$

и используя соотношения ортогональности для базисных элементов

$$
\begin{aligned}
\left\langle T_{i}^{n}, T_{j}^{m}\right\rangle & =2\left(h_{i}, h_{j}\right) \delta_{n, m}, & & n, m>0, \\
\left\langle W_{\alpha}^{n}, W_{\beta}^{m}\right\rangle & =2\left(e_{\alpha}, e_{-\alpha}\right) \delta_{\alpha \beta} \delta_{n, m}, & & n, m>0,
\end{aligned}
$$

находим явный вид оператора $r$ (общий множитель $1 / 2$ опущен):

$$
r=\sum_{\substack{k>0 \\ 1 \leq i, j \leq l}} a^{i j} H_{i}^{k}\left\langle T_{j}^{k}, \ldots\right\rangle+\sum_{\alpha \in \Delta_{+}} V_{\alpha}^{0}\left\langle W_{\alpha}^{0}, \ldots\right\rangle+\sum_{\substack{k>0 \\ \alpha \in \Delta}} V_{\alpha}^{k}\left\langle W_{\alpha}^{k}, \ldots\right\rangle,
$$

где $a^{i j}$ - матриша, обратная к $\left(h_{i}, h_{j}\right), 1 \leq i, j \leq l$. Этот оператор определяет следующий элемент пространства $\mathcal{K} \otimes \mathcal{M}$, которьй мы также обозначаем буквой $r:$

$$
r=\sum_{\substack{k>0 \\ 1 \leq i, j \leq l}} a^{i j} H_{i}^{k} \otimes T_{j}^{k}+\sum_{\alpha \in \Delta_{+}} V_{\alpha}^{0} \otimes W_{\alpha}^{0}+\sum_{\substack{k>0 \\ \alpha \in \Delta}} V_{\alpha}^{k} \otimes W_{\alpha}^{k}
$$

Заметим, что $r$-матрица как вектор пространства $\mathcal{K} \otimes \mathcal{M}$ определена неоднозначно. Поскольку в (20) элементы $L, X, Y \in \mathcal{M}$, к (24) всегда можно добавить произвольный 
вектор из $\mathcal{M} \otimes \mathcal{K}$, не изменив при этом значения скобки. Таким образом, можно ввести оператор $R=r-\sigma(r)$, где $\sigma$ есть оператор перестановки в $\mathcal{G} \otimes \mathcal{G}$. Подставляя в $R$ определения образуюших (5), получаем

$$
\begin{aligned}
R^{\mathrm{st}}= & \sum_{\substack{k>0 \\
1 \leq i, j \leq l}} a^{i j}\left(h_{i}^{k} \otimes h_{j}^{-k}-h_{i}^{-k} \otimes h_{j}^{k}\right)+\sum_{\alpha \in \Delta_{+}}\left(e_{\alpha}^{0} \otimes e_{-\alpha}^{0}-e_{-\alpha}^{0} \otimes e_{\alpha}^{0}\right)+ \\
& +\sum_{\substack{k>0 \\
\alpha \in \Delta}}\left(e_{\alpha}^{k} \otimes e_{-\alpha}^{-k}-e_{-\alpha}^{-k} \otimes e_{\alpha}^{k}\right),
\end{aligned}
$$

где $R^{\text {st }}$ означает $r$-матрицу в стандартной градуировке. Построенная таким образом $R^{\text {st }} \in \mathcal{G} \otimes \mathcal{G}$ совпадает с классической тригонометрической $r$-матрицей модели Тоды, соответствующей стандартной градуировке $L$-оператора (19). В самом деле, отождествляя $\mathcal{G} \otimes \mathcal{G}$ с $\mathcal{G}\left[\lambda, \lambda^{-1}\right] \otimes \mathcal{G}\left[\mu, \mu^{-1}\right]$, т.е. подставляя в (25) стандартную реализацию образуюших и выполняя суммирование, находим

$$
R^{\mathrm{st}}=\frac{\lambda r_{-}-\mu r_{+}}{\lambda-\mu},
$$

где

$$
r_{+}=\frac{1}{2} a^{i j} h_{i} \otimes h_{j}+\sum_{\alpha \in \Delta_{+}} e_{\alpha} \otimes e_{-\alpha}, \quad r_{-}=-\sigma\left(r^{+}\right)
$$

суть два константных решения классического уравнения Янга-Бакстера.

2. $r$-Матрица в канонической градуировке. Вычисления в канонической градуировке абсолютно аналогичны случаю 1 , поэтому мы приведем только ответы для элементов $r$ и $R^{\text {can }}$ соответствующих $r$-матриц в канонической градуировке:

$$
\begin{aligned}
r= & \sum_{\substack{k>0 \\
1 \leq i, j \leq l}} a^{i j} H_{i}^{k h} \otimes T_{j}^{k h}+\sum_{\substack{k>0 \\
k \neq 0 \bmod h}} \sum_{\alpha \in \Delta^{k \bmod h}} V_{\alpha}^{k} \otimes W_{\alpha}^{k}, \\
R^{\mathrm{can}}= & \sum_{\substack{k>0 \\
1 \leq i, j \leq l}} a^{i j}\left(h_{i}^{k h} \otimes h_{j}^{-k h}-h_{i}^{-k h} \otimes h_{j}^{k h}\right)+ \\
& +\sum_{\substack{k>0 \\
k \neq 0 \bmod h}} \sum_{\alpha \in \Delta^{k \bmod h}}\left(e_{\alpha}^{k} \otimes e_{-\alpha}^{-k}-e_{-\alpha}^{-k} \otimes e_{\alpha}^{k}\right) .
\end{aligned}
$$

Вьполняя в последней формуле суммирование канонических образуюших, получаем хорошо известное [1] решение классического уравнения Янга-Бакстера, отвечаюшее канонической градуировке алгебры $\mathcal{G}$ :

$$
R^{\text {can }}=\frac{t_{0}}{2}+\frac{1}{x^{h}-1} \sum_{k=0}^{k=h-1} t_{k} x^{k},
$$

где $x=\lambda / \mu, t_{0}=a^{i j} h_{i} \otimes h_{j}$ и $t_{k}=\sum_{\alpha \in \Delta^{k}} e_{\alpha} \otimes e_{-\alpha}$. 
Таким образом, метод гамильтоновой редукции, надлежащим образом примененный к кокасательному расслоению над группой петель, позволяет строить $r$-матрицы, зависящие от спектрального параметра. Подчеркнем, что сама возможность редукции обусловлена наличием разложения Ивасавы $G=N_{+} A K$, в котором группа $A$ оказывается конечномерной, так что конечномерным оказывается и редуцированное фазовое пространство. Описанная ситуация уже не имеет места для разложения Картана $G=K A K$ комплексной группы петель $G$, т.е. фактор $A$ оказывается бесконечномерным. Заметим, что в конечномерном случае указанное разложение Картана участвует в гамильтоновой редукции $\mathcal{K} \backslash T^{*} G / \mathcal{K}$, приводящей к моделям Калоджеро-Мозера-Сазерленда [3]. По-видимому, $r$-матришы со спектральным параметром для этих моделей должны получаться методом гамильтоновой редукции $T^{*} G(\lambda)$, в котором вместо группы $\mathcal{H}=\mathcal{K} \times \mathcal{K}$ используется какая-то другая группа диффеоморфизмов группы петель $G(\lambda)$. Мы надеемся вернуться к рассмотрению этого вопроса в последуюших публикациях.

Автор благодарен А. Горскому, П. Медведеву и С. Фролову за полезные обсуждения. Работа выполнена при финансовой поддержке РФФИ, грант N96-01-00608.

\section{Список литературы}

[1] А. А. Белавин, В. Г. Дринфельд. Функц. анализ и его прилож. 1982. Т. 16. № 3. С. 1-29.

[2] M. Jimbo. Springer Lectures in Physics. In: Current Topics in QFT. Seminar Proceedings. Berlin: Springer, 1985. P. 35-62.

[3] M. A. Olshanetsky, A. M. Perelomov. Invent. Math. 1976. V. 37. P. 93.

[4] L. Ferreira, D. Olive. Commun. Math. Phys. 1985. V. 99. P. 365-384.

[5] J. Avan, O. Babelon, M. Talon. Алгебра и анализ. 1994. Т. 6. № 2. С. 67-89.

[6] Л.А. Тахтаджкян, Л.Д. Фаддеев. Гамильтонов подход в теории солитонов. М.: Наука, 1986.

[7] В. Кац. Бесконечномерные алгебры Ли. М.: Мир, 1993.

[8] Э. Прессли, Г. Сигал. Группы петель. М.: Мир, 1990.

[9] В. И. Арнольд. Математические методы классической механики. М.: Наука, 1989.

[10] O. I. Bogoyavlensky. Commun. Math. Phys. 1976. V. 51. P. 201-209.

Поступила в редакцию 30.V.1997 г.

\section{G.E. Arutyunov \\ CONSTRUCTION OF TRIGONOMETRIC TODA $r$-MATRICES VIA HAMILTONIAN REDUCTION OF THE COTANGENT BUNDLE OVER LOOP GROUPS}

By applying the Hamiltonian reduction method to the cotangent bundle over loop groups, we recover the well-known classical trigonometric $r$-matrices of the periodic Toda lattice. 